the knowledge and skill they profess must be protected against those who only pretend to have acquired that skill. The public who are asked to pay for the care they need in sickness, whether the rate of charge be low or at the customary rate for skilled service, must be protected also from the same evil. And what is of still greater importance, the nursing profession itself must be protected against this great body of women of inferior breeding, education and training, who, whether they undertake domestic or private nursing, pose as nurses, and by their ignorance lower its standards in the eye of the public.

When our pupils are trained from the beginning with the view of entering for state examinations at the end of their course in the hospital, the best women will qualify themselves, first, by securing a good general education, and then enter only the schools where the highest standards obtain. This will do much toward eliminating irregular schools, and with them the incompetent, untrained or poorly trained nurse.

\title{
THE CARE OF THE BREASTS IN OBSTETRICAL CASES
}

\author{
BY KATHARINE DE WITT \\ Graduate Illinois Training-School for Nurses
}

\section{PREFATORY NOTE}

IT is the desire of the Editor of the Private Nursing department to receive, for use in this place, papers on practical nursing subjects which shall be not scientific treatises, but accounts of cases, describing the nursing care given to patients from the nurse's point of view. There are undoubtedly many private duty nurses who are deeply interested in their work and who are full of ideas concerning it, who would yet hesitate to write articles on their work for fear they might have nothing new or original to offer. Two facts should be borne in mind. First, if we wait to hear from gifted and brilliant women, we shall have very few, if any, papers; for the great majority of us are possessed of only ordinary ability and can, perhaps, enjoy ordinary papers better than too learned ones. Second, when we consider the hundreds of nurses, all over the world who read the Journal, we are safe in believing that, hardly a suggestion can be made which will not prove new and helpful to some one. In the article following, no new ideas are advanced what is written will be an old story to many, but it is hoped that some one who reads it will say to herself, "Why, I know a better way than that!" and that she will not only think this but will share her wider knowledge with us, that all may be benefitted. If the articles published in this department can be thus made a starting-point for questions, answers, and suggestions,all of which will be considered under the heading. Practical Points,- the exchange of ideas will be helpful to us all. 
l'he cure of the breasts and mipples should begin in youth, as too great a pressure from corsets or from tightly-fitting undergarments may, sometimes, result in tlattened or depressed nipples. 'This hardly comes within the oversight of a nurse, however, except in the way of advice to mother's regiarding their young daughters; and fortunately the present mode in corsets supplies a very low bust which is no support at all, and which certainly camnot make undue pressure. During pregnancy the breasts of a patient are examined by her doctor, and if he finds the nipples inverted or Hat, he usually tells lier to manipulate them gently, once or twice a day, drawing them out as much as possible. This liandling of the nipples makes some women very nervous, or even nauseated, and in such cases it should be discontinued, as most bad nipples can be improved later by means of a nipple shield. Formerly, different washes for toughening the nipples and preparing them for nursing, were much used, such as alcohol, alcohol and water, a solution of tannic acid in glycerine, etc. Now, most physicians advise the patient simply to keep the nipples perfectly clean by means of gentle bathing. If crusts form on the surface, they may be softened by using some emollient,- white vaseline, albolene, or cocoa butter. Every well-trained nurse is taught that, from the moment a child is born, the breasts and nipples are to be kept in as aseptic a condition as possible. The breasts should be covered with a broad strip of sterile gauze, ample enough to protect the whole breast surface, over this is fastened the breastbinder. The breast-binder is a familar article to most obstetrical nurses, but $I$ have occasionally met one who was not accustomed to the kind commonly used, or who thought a towel with shoulder straps just as good. It should be made in this shape, and is far more comfortable than a towel. The long part of the binder is placed under the back, drawn under the arms, and is pinned in front, the breasts, meanwhile, being lifted and held in place by the patient. The shoulder-pieces are pinned over after the rest is properly adjusted, and need not then be unpinned again until the binder is removed at bath time. At nursing. time all the pins in front are removed, and the gauze is carefully rolled under and away from the breast to be nursed, not carelessly brushed aside to collect stray germs and carry them back to the nipple again. The patient must be taught how to do this, and must be carefully instructed by the nurse, as soon as she is strong enough to listen, not to touch the nipple with her fingers and not to allow anything to brush against it when it is uncovered. When a baby is brought to its mother, she is apt to forget all precautions, and the baby's shoulder, with its covering of knit blanket, is drawn across the nipple. The nurse must 
always be on the watch to prevent such accidents, for an ounce of precaution in these respects is worth a pound of cure later. The usual method of treating the nipple is to wash it with boracic acid solution before and after each nursing. The best way of applying this is by an applicator, a tooth-pick wound with cotton. Several hundred of these applicators should be prepared before the birth of the baby, and should be done up in small packages and carefully sterilized. A jelly glass, with a glass or tin cover, should also be boiled and carefully wrapped and put away, ready to receive them when the time comes for their use. The boracic acid solution is contained in a small, sterile, wide-mouthed bottle. By using applicators, instead of sponges, and by being careful never to touch the cotton part with the fingers, many chances of infection are excluded. A nurse should never, never change a baby's diapers and then proceed to prepare the breast for nursing without first thoroughly washing the hands. As a rule, the baby's mouth is let alone, but a few physicians still prefer to have it washed before a nursing. This must be done gently, as it is easy to cause an abrasion on che mucous membrane, which is painful to the child and a starting point for trouble.

The engorgement of the breasts on the third day, which often occurs, and is so painful, can sometimes be prevented by limiting the amount of liquids given during the first few days. The taking of much milk at this time will not hasten the arrival of the milk, but may aggravate the discomfort of the patient when it does come. This first engorgement, however, is not wholly caused by a flood of milk, but also by distended veins and lymphatics. For this reason, the use of a breast-pump relieves the patient only a little. A very firm breast-binder is the most rational method of relief for a distension which will soon pass away of its own accord. Even after nursing is well established, different portions of the breasts may at times seem hard. If the hard part is stroked gently while the baby nurses, it will usually become soft.

The nipples are almost always sensitive for the first few days; they have to grow accustomed to their new task; and they are often particularly painful before the milk supply is established. For this reason, it is better not to put the baby to the breast too often a first, and not to let it remain too long; a nursing. once in six hours is enough to keep up the nursing habit in the baby, and from three to five minutes is a sufficient length of time for it to remain. By the middle of the second day, the interval can be reduced to four hours, and when the milk appears, to two and a-half or two, whichever is to be the established period. A two-and-a-half-hour interval between meals by day, and a 
four-hour interval at night, give eight meals in the twenty-four hours, which are enough for a normal baby, and give the mother more rest than the two-hour intervals do. The milk supply depends, for its continuance, upon good health in the mother, and upon her having wholesome food at regular intervals and in proper amounts, plenty of sleep, and a quiet mind. Sleep is a most important factor. A mother who is wakened every two hours all night to nurse her baby, is too tired to produce a good food, and the baby may be wakeful and fretting on account of the deterioration in quality, which could be remedied by giving it hot water to drink, and letting the mother have the rest which is so needful for the good of both. To overload the mother's stomach with all sorts of liquids, many of which are distasteful to her, is a method of upsetting her digestion without aiding the milk supply. All good, wholesome food helps it, though probably milk, cocoa, cereals, and gruels are a more direct aid. Many kinds of food which are not directly useful to the baby are so to the mother as an aid to her appetite, which must be carefully watched, as it is easily sated by a preponderance of milky viands. Foods which disagree with the mother in health must, of course, be omitted, but fresh ripe fruits, in season, can usually be given safely, and simple salads with a French dressing are a wholesome as well as an appetizing addition to the diet. Lemon and orange juice do not affect the milk badly, but very strongly flavored vegetables do, such as onions, cabbage and cauliflower. Most vegetables are useful, particularly carrots and beets. A nursing mother can usually take three good meals a day, with additional drink in the night. Many like also the morning or afternoon glass of milk, and this will digest better if crackers are given with it, but none should be given when the patient feels that to take it will be an effort. In providing a diet for a wetnurse, a great many people make the mistake of giving her too delicate food. She is not used to it and longs for the heartier and plainer fare to which she is accustomed and on which she will really thrive better.

To go back to the actual question of nursing. The position of the baby at the breast is a most important one. When the mother can sit up and hold the baby, she can usually adjust it comfortably, but when lying down she cannot judge as well how it lies, and often gets its head at a distressing angle, or has its body too low, so that the baby is reaching up for its dinner. In such a condition the baby will stop nursing from sheer fatigue before its appetite is appeased. The most comfortable position for the baby is to lie flat on the bed, the mother turning toward it to nurse it, but this proves tiresome to some women, as the arm nearest the baby has to be held up out of the way. If the baby 
lies on the mother's arm to nurse, which is a more natural position, the nurse must see that it is properly held to begin with, and must look at it occasionally to adjust it, if it is slipping away from the breast. A pillow tucked at the mother's back is a great relief to her, for she often holds herself in a strained position without realizing it, and is tired when the nursing is ovcr. Toward the end of a nursing, or when the baby is not very hungry, it often slips away a little and bites on the end of the nipple. This is always painful and increases the sensitiveness of the nipple. The patient must be urged to tell when such " nipping" begins, for therc is no virtue in bearing it heroically, as the baby is getting no food when doing this. The nurse must put her fingcr gently between the baby's jaws at the back of the mouth and remove it entirely from the nipple. If still hungry, it will soon take a new and better hold. If it is always rcmoved when it nurses badly, it will soon learn to do better.

If the nipples are very scnsitive and painful at first, or if an abrasion appears, a nipplc-shicld of glass and rubber, of the simplest pattern, should be used. This should be thoroughly washed and boiled before being applied, and a finger-bowl containing boracic acid solution, enough to wholly cover it, should be in readiness to hold it after it has been used and washed thoroughly. In order that the nipple-shield may work well, the rubber tip must fit very tightly orer the glass, and the glass part must be held by the mother tightly against the breast. Sometimes it is necessary to wind the rubber part with silk thread to bind it tightly, though it is thus rendered difficult to clean. The shield should be filled with sterile water before being applied, and the mother must turn toward the child in such a way that it will be pulling straight and keeping its mouth directly against the little bone shield which gives it a brace. If the shield is put on empty and the milk is hard to start, a listless baby will become discouraged and will not suck after the first few fruitless attempts, but the shield filled with warm water usually gives it courage to go on. and the milk, as a rule, begins to come before the water is gone. If the milk is very slow in starting, and the baby feeble or reluctant, hot sterile cloths can be applied over and around the nipple, for five minutes before the nursing, after which the milk comes easily; or a breast pump may be used just long enough to start the milk and to draw enough to fill the shield. This meth od may be tried, also, when a haby refuses to nurse from the breast, as sometimes happens. As a rule the nipple-shield need not be used through the wholc nursing, except where there is an abrasion on the nipple to be protected. If the nipple is merely sensitive or is flat so that the baby cannot grasp 
it unaided, five or ten nimutes' nursing with the shicld will be enough and the baby can then be put directly to the breast to finish its meal. When a patient has a very abundant milk supply, and the baby eats too fast, getting its whole mcal in a few minutes and then crying from discomfort, the nipple-shield is of grcat usc. If put on at the beginning of a nursing, during the first rush of the milk, the baby will get all it needs, but witl greater moderation and comfort. Lead nipple-shields, which are not perforated, are often a great comfort in preventing any pressure or rubbing from the clothing. These, also, are first washed and boiled and are then put over the nipples, held in place by the binder, and are worn all the time between nursings.

If a fissure, however tiny, appears on the surface of the nipple, all precautions must be taken to guard it from infection and to prevent its becoming enlarged. The doctor will usually touch it with a weak solution of nitrate of silver or will order the nurse to do so, and the baby will either be taken from that breast for a time or nursed from it at longer intervals. A fissure will heal quickly with rest and care, unless the nipple is badly shaped, when it may be very obstinate.

If a small red spot, feeling sensitive to the touch, appears anywhere on the breast surface, the doctor must be notified at once, especially if the patient has also a chill and rise of temperature. If the trouble seems merely local, treatment by hot compresses, if instituted promptly, will often relieve it. A compress of linen is boiled to make it sterile and is wrung out of hot water, (not too hot to be borne by the hands), is put over the sensitive place and covered with oiled silk and flannel. The binder is then pinned as usual, and the compress is left alone until the next nursing, when another cloth is boiled to replace the first one. If the doctor fears mastitis he will order a saline cathartic for the patient; and for local treatment, either regular fomentation: changed every few minutcs, or ice-bags. If ice is used, the patient feels chilly at first, until she is used to them, and she must be kept warm with hot-water bags. She will sometimes need one at her feet while the treatment lasts, from twenty-four to forty-eight hours. The baby, in this case, cannot nurse from the affected breast until the trouble is over, and must be fed from a bottle, alternately with nursing from the other side. When the ice-bags are removed, the milk must be taken from the breast with a pump and discarded, as it will not agree with the baby.

If the patient is a cleanly woman, and the nurse is conscientious and faithful in her care, there should be no chance of external infection. Possibly inflammation may arise from conditions within the breasts, themselves, but the nature of it is not as yet clear. 